\title{
Low-energy Electronic Excitations in Transition-metal Oxide as Probed by STEM-EELS Spectromicroscopy
}

Alexandre Gloter ${ }^{1}$, Chiaping $\mathrm{Su}^{1}$, Xiaoyan $\mathrm{Li}^{1}$, Kari Ruotsalainen ${ }^{2}$, Alessandro Nicolaou ${ }^{3}$ and Odile Stephan $^{1}$

${ }^{1}$ Université Paris-Saclay, CNRS, Orsay, Ile-de-France, France, ${ }^{2}$ Helmholtz-Zentrum Berlin für Materialien und Energie, Berlin, Berlin, Germany, ${ }^{3}$ Soleil Synchrotron, Gif-sur-Yvette, Ile-de-France, France

The transition metal (TM) oxides, e.g. TM 3d-based oxides with perovskite related structures, exhibit a plethora of properties such as superconductivity, ferro-electricity, magnetism, etc., Indeed, beside the atomic structural degree of freedom, the electrons have internal degrees of freedom, such as charge, spin, and orbital, leading to various forms of ordered states through phase transitions. Furthermore, the coupling between these orders can be strong in these systems leading to additional properties (multiferroism), or the possibility of controlling these orders by external stimuli (e.g., ferroelectric tunnel junctions [1]) or nano-structuration (e.g., strain or interfacial magnetism [2]). All these orders give collective excitations (phonon, plasmon, magnon, orbiton) possibly studied, for instance, by resonant or non-resonant inelastic X-ray scattering (IXS), low-energy spin-polarized electron energy-loss spectroscopy EELS or inelastic neutron scattering.... Since the arrival of monochromator in scanning transmission electron microscopy (STEM) with an energy resolution down to $5 \mathrm{meV}$ [3], such excitations might also, to some extent, be investigated by high-energy transmission EELS. Here we will focus on the low energy electronic excitations of the transition-metal oxide, id est, the plasmon, the metal-metal (d-d) and charge-transfer (pd) excitations and discuss how they can be related to the electronic structure of nanostructures.

We will first discuss how the dipole forbidden d-d transitions are observable in EELS [4]. For transitionmetal monoxide ( $\mathrm{MnO}, \mathrm{NiO}, \mathrm{CoO}$, etc..) such excitations have been well characterized by IXS [5] but many questions still reside when investigated by EELS (selectivity in term of multipolar transition ? how atomic are their characters ? effectiveness of $\omega(\mathrm{q}, \mathrm{r})$ measurements with Cs corrected/monochromated STEM). We will then discuss the observation of $\mathrm{d}-\mathrm{d}$ based plasmons in the case of metallic $3 \mathrm{~d}$ based transition-metal oxide, e.g. $\mathrm{SrVO}_{3}$. It is indeed fascinating that TM-3d bands can result in such different electronic excitations as observed by EELS. The figure 1 clearly evidences this difference of behaviour between atomic type $\mathrm{d}$-d excitations of $\mathrm{NiO}$ (no dipolar contribution, no observable q-dispersion within the EELS accuracy, no surface delocalisation in aloof geometry) and a plasmonic type d-d excitations of $\mathrm{SrVO}_{3}$ (dipolar contribution, q-damping and small q-dispersion, surface plasmon in aloof geometry, ...) [6].

One important aspect of the d-d excitations concerns their spatial resolution, notably if one aims at revealing by STEM-EELS the coupling between structure and TM-d electronic structure reconstruction in TM oxide heterostructures. Figure 2 shows how the non-dipolar d-d transitions evolve at a nanometer scale when crossing the interface between a $\mathrm{NiO}$ and a $\mathrm{NdNiO}_{3}$ nanocrystals, revealing different $\mathrm{Ni}-\mathrm{d}$ ground states. On the other hand, nanostructured $\mathrm{SrVO}_{3}$ result in a Fabry-Perot type d-d based surface plasmon polariton modes. In this case, the spatial/energy distribution relates more with the geometry of the nanostructure (e.g. particle aspect ratio) than the ground state evolution of the TM $3 \mathrm{~d}$ band.

We thank Matteo Gatti (CNRS-Ecole Polytechnique), Mathieu Kociak (CNRS-Université Paris-Saclay), Guillaume Radtke (CNRS-Sorbonne Université), Luiz Tizei (CNRS-Université Paris-Saclay) and Alberto Zobelli (CNRS-Université Paris-Saclay) for fruitful discussions. This work has received support from the 
National Agency for Research under the program of future investment TEMPOS CHROMATEM with the Reference No. ANR-10-EQPX-50.

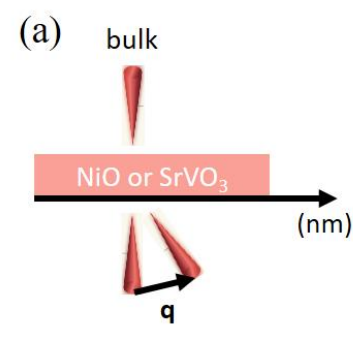

(b)

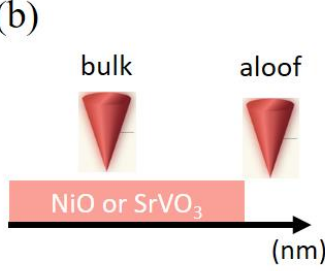

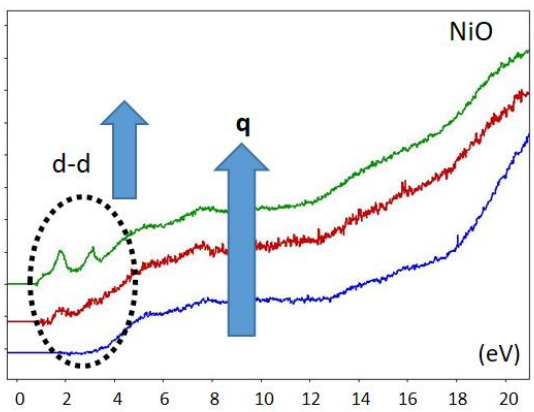

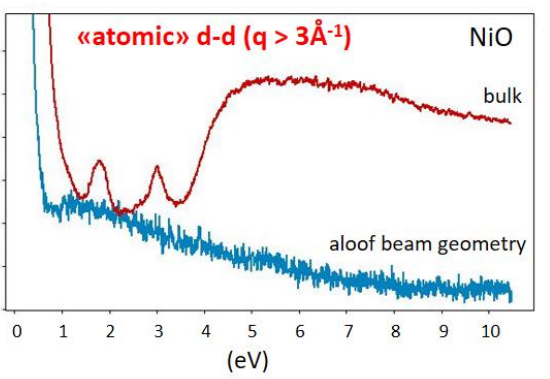

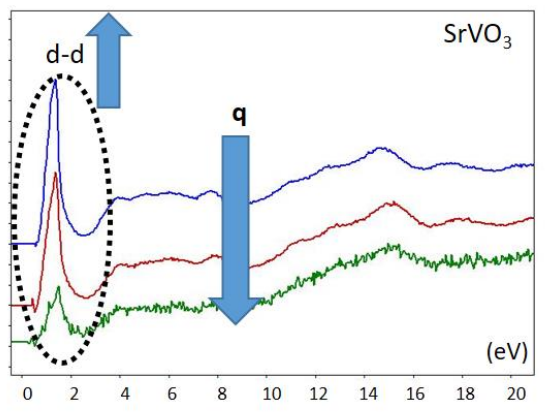

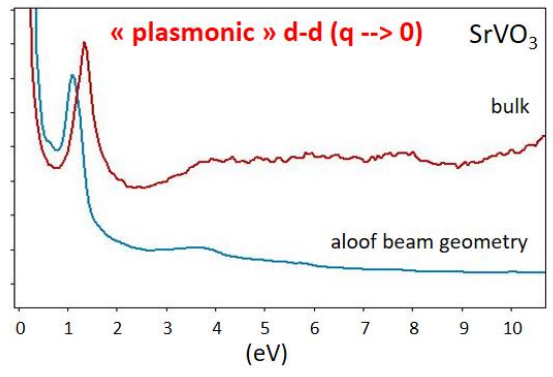

Figure 1. (a) Dispersion curve and (b) bulk/aloof EELS measurements of NiO and SrVO3.
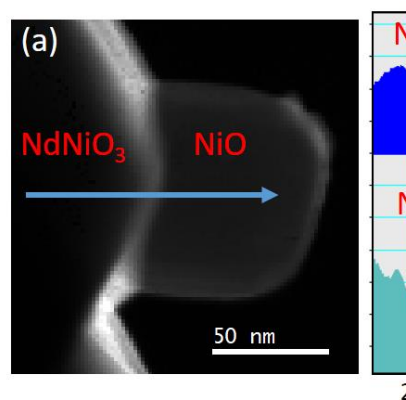
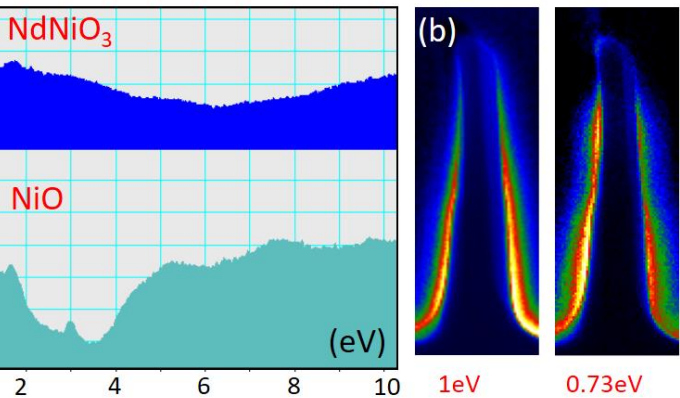

$0.73 \mathrm{eV}$

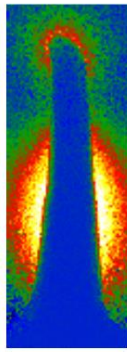

$0.50 \mathrm{eV}$

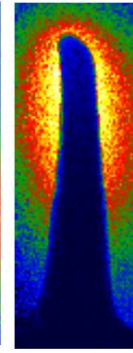

$0.24 \mathrm{eV}$

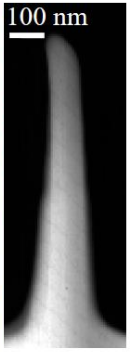

$\mathrm{SrVO}_{3}$

Figure 2. (a) Non-dipolar EELS measurements from $\mathrm{NiO} / \mathrm{NdNiO} 3$ heterostructure, (b) spatial distribution of plasmonic modes in a SrVO3 nanostructure.

\section{References}

[1] V. Garcia et al., Ferroelectric Control of Spin Polarization, Science. 327, 1106-1110 (2010)

[2] M. Gibert et al., Interfacial Control of Magnetic Properties at $\mathrm{LaMnO}_{3} / \mathrm{LaNiO}_{3}$ Interfaces, Nano Lett. 15, 7355-7361 (2015)

[3] O.L. Krivanek et al., Vibrational spectroscopy in the electron microscope, Nature 514, 209-212 (2014) [4] A. Gloter et al., Probing non-dipole allowed excitations in highly correlated materials with nanoscale resolution, Ultramicroscopy 109, 1333-1337 (2009)

[5] M.W. Haverkort et al., Nonresonant inelasticX-ray scattering involving excitonic excitations: the examples of $\mathrm{NiO}$ and $\mathrm{CoO}$, Phys. Rev. Lett. 99, 257401-257 (2007)

[6] In preparation 